\title{
Inflammatory abdominal aortic aneurysm: a case report
}

\author{
AVDLA Vithanage ${ }^{1}$, MCB Galahitiyawa ${ }^{1}$, JDVC Lekamwasam ${ }^{2}$, Sarath Lekamwasam ${ }^{3}$ \\ ${ }^{1}$ Registrar in Medicine; ${ }^{2}$ Consultant Physician, Teaching Hospital, Karapitiya, Galle. \\ ${ }^{3}$ Professor in Medicine, Department of Medicine, Faculty of medicine, University of Rununa, Galle.
}

A 74 year-old, retired clerk was having lower backache and loss of weight for one month. Pain, at times, radiated to both loins and testicles. It was aggravated by walking but not relieved by any particular position. He had nausea but not vomiting, dysuria or frequency. His bowel habits were normal. He had been smoking 4 to 5 cigars per day for nearly 20 years and consumed alcohol occasionally.

On admission he was in pain. He was not pale or icteric. No cervical or supraclavicular lymph nodes were palpable and no finger clubbing was detected. Clinical examination of the abdomen and lungs were normal. His initial blood pressure and pulse rate were 190/100 $\mathrm{mmHg}$ and $80 \mathrm{bpm}$. He had normal heart sounds. His lumbar spine was non-tender and the strait leg raising (SLR) test was normal.

Initial investigations were as follows.

He had haemoglobin of $10 \mathrm{~g} / \mathrm{dL}$, WBC of 14800 $/ \mathrm{mm}^{3}$ with $92 \%$ neutrophils. Normochromic and normocytic red cells with increased roulex formation was seen. ESR was $126 \mathrm{~mm}$ in $1^{\text {st }}$ hour and CRP was $26 \mathrm{mg} / \mathrm{L}$. Serum creatinine, blood urea and liver transaminases were within normal limits. The first ultrasound scan abdomen found only prostatomegaly and his PSA was within normal limits. Lumbo-sacral radiograph was reported normal and free of malignant secondaries. His sputum for AFB was negative thrice and Mantoux reaction was normal. Skeletal survey and serum electrophoresis were unremarkable.

In the third week of admission, a pulsating abdominal mass was found during clinical examination and ultrasonically it was found to be an abdominal aortic aneurysm (AAA), $6 \mathrm{~cm}$ wide and $7 \mathrm{~cm}$ long. There was a thrombus in the left lateral wall. The aneurysm was arising just below the origin of the renal artery and extending up to the bifurcation of the aorta. Contrast enhanced CT abdomen confirmed the AAA (Fig. 1). Patient was transferred to the Vascular Unit of the National Hospital, Colombo for further management. Patient died of aneurysmal rupture one week later.

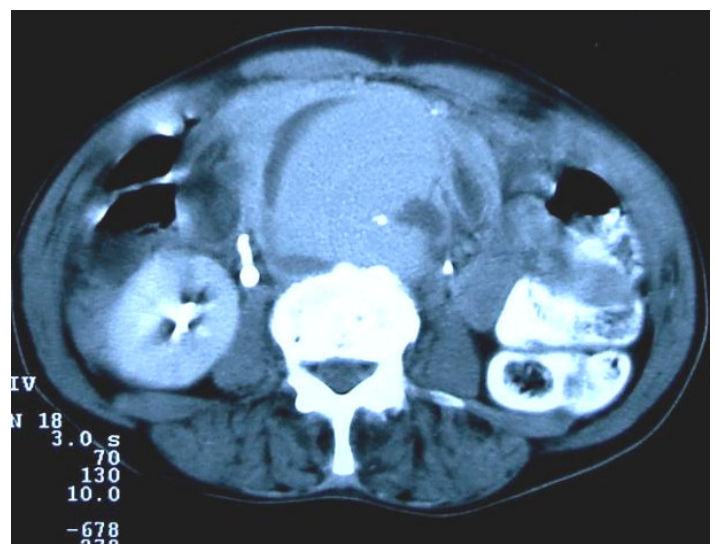

Fig. 1 - Contrast enhanced CT scan of abdomen showing aortic aneurysm with large clot within the lumen.

\section{Discussion}

Abdominal aorta is the most common site of aneurysmal dilatation. The prevalence of AAA is negligible below the age of 60 . It is 4 to 5 times commoner in men. According to screening studies, 4 to $6 \%$ of adults have an AAA. However, most of these aneurysms are less than $3.5 \mathrm{~cm}$ in size and clinically not significant ${ }^{1}$.

Clinically significant aneurysms are either symptomatic or greater than $5.5 \mathrm{~cm}$ in size. Most aneurysms are asymptomatic and remain quiescent until rupture. Hence AAAs which are less than $5.5 \mathrm{~cm}$ in size and asymptomatic should be followed up with serial abdominal USS. 
Pathogenesis of AAA remains poorly understood. Two main types are atherosclerotic and inflammatory. They have different clinical presentations, histopathology and prognosis. Recent studies suggest a common aetiopathogenic mechanism for both types of aneurysms. Environmental factors such as tobacco use may induce chronic inflammation in some people predisposing them to inflammatory AAAs (IAAA) ${ }^{2}$. Around $5 \%$ of all aneurysms are inflammatory.

The clinical presentation of IAAA is low back pain, abdominal pain, loss of weight and high ESR. Abdominal palpation may show transmitted or expansile pulsation and tenderness. These features indicate recent aneurysmal expansion and harbinger of rupture. At operation marked thickening of aneurysmal wall, dense adhesions around and occasional retroperitoneal fibrosis are seen. Entrapment of the adjacent organs in the fibrotic process is the main complication. Ureteric and duodenal obstruction with consequent hydroureteronephrosis and bowel obstruction have been reported ${ }^{3}$. Hence surgical repair of IAAA is a challenge. Contrast enhanced CT abdomen and gadolinium enhanced MRI are highly sensitive methods in diagnosing IAAA. USS abdomen has less sensitivity.

Operative repair and insertion of a prosthetic graft is indicated in IAAA. Endovascular repair and stent placement is the emerging approach but has a mortality rate of $40 \%$. A study has shown that accurate diagnosis and careful pre and postoperative management of IAAA has similar survival rate to that of $\mathrm{AAA}^{4}$.
Some believe initial corticosteroids are useful in IAAA with severe inflammation or ureteric involvement ${ }^{5}$. Due to regression of fibrosis it may help subsequent surgery.

\section{References}

1. Singh K, Bonaa KH, Jacobsen BK, et al. Prevalence of and risk factors for abdominal aortic aneurysms in a population-based study: The Tromso Study. American Journal of Epidemiology 2001; 154: 236-44.

2. Rasmussen TE, Hallett JW. Inflammatory aortic aneurysms. A clinical review with new perspectives in pathogenesis. Ann Surg. 1997; 225(2): 155-64.

3. Scuro A, Barzaghi ME, Griso A, Ferrari Ruffino S, Kontothanassis D, Mirandola M, Leonardi G, D'Agata M. Approach to Juxtarenal inflammatory aneurysms. Ann Ital Chir. 2004; 75(2): 199-209.

4. Sasaki S, Yasuda K, Takigami K, Yamauchi H, Shiiya N, Sakuma M. Inflammatory abdominal aortic aneurysms and atherosclerotic abdominal aortic aneurysms-comparisons of clinical features and longterm results. Jpn Circ J. 1997; 61(3): 231-5

5. Testart J, Plissonnier D, Peillon C, Watelet J.Inflammatory abdominal aortic aneurysm. Role of corticosteroid therapy. J Mal Vasc. 2000; 25(3): 2017. 